\title{
A Case Report of Classical Gastrointestinal Stromal Tumor with an Undifferentiated Pleomorphic Sarcoma Component
}

\section{YiFan $\mathrm{Hu}$}

Affiliated Hospital of North Sichuan Medical College https://orcid.org/0000-0001-7069-451X

SHUNHAI JIAN (D2051084695@qq.com )

Affiliated Hospital of North Sichuan Medical College https://orcid.org/0000-0002-5940-1365

bin wen

Affiliated Hospital of North Sichuan Medical College

linli he

Affiliated Hospital of North Sichuan Medical College

yifan huang

Affiliated Hospital of North Sichuan Medical College

\section{Case Report}

Keywords: Gastrointestinal stromal tumor(GIST), Imatinib (IM), undifferentiated pleomorphic sarcoma

Posted Date: September 17th, 2020

DOl: https://doi.org/10.21203/rs.3.rs-55392/v1

License: (9) This work is licensed under a Creative Commons Attribution 4.0 International License. Read Full License 


\section{Abstract}

Background:Gastrointestinal stromal tumor (GIST) is the most common primary mesenchymal neoplasm of the gastrointestinal tract.a small number of GIST patients showed morphologic and immunohistochemical phenotypes change after taking the selective tyrosine kinase inhibitor imatinib.

Case presentation :A 64-year-old Asian man was referred to a hospital for dizziness and anemia. Then the patient received imatinib for 11 months after the discovery of a gastric stromal tumor, and had the tumor surgically removed. We found that the classical GIST region was juxtaposed to a undifferentiated component of pleomorphic sarcoma. The genetic test results of the patient showed that the 11 exon of CKit gene was homozygous deletion, and the 567 codon of the 12 exon of PDGFRa was homozygous mutation. We infer that the patient showed rare dedifferentiation change after taking the tyrosine kinase inhibitors (TKIs) imatinib囚IM区.

Conclusions:Together with reported cases, awareness of this rare clinical entity and its potential occurrence following Tyrosine kinase inhibitor (TKI)treatment could prevent a diagnostic pitfall. The case report reminds us of the diagnosis in GIST that the possibility of dedifferentiation in GISTs should always be considered when an undifferentiated sarcoma component is identified in the gastrointestinal tract.

\section{Background}

Gastrointestinal stromal tumor is the most common mesenchymal tumor of the gastrointestinal tract. It originates from the gastrointestinal mesenchymal cells of Cajal and can occur in any part of the whole digestive tract, mainly in the stomach, small intestine, colorectal and esophagus.[1]Epidemiological studies show that the age of onset of gastrointestinal stromal tumors ranges from 66 to 69 years old, but $75 \%$ of patients with symptoms of GIST are over 50 years old. There is no obvious boundary between benign and malignant gastrointestinal stromal tumors, and the degree of benign and malignant is different .At present, the KIT gene and PDGFRa gene mutations in the patients with somatic cell is considered to be its main driving factors in the development of GISTs[2].

Clinically, a small number of GIST patients showed morphologic and immunohistochemical phenotypes alteration after taking the selective tyrosine kinase inhibitor imatinib, and the tumor underwent dedifferentiation process. The current reports include rhabdomyosarcoma, angiosarcoma and undifferentiated pleomorphic sarcoma. The dedifferentiated component, which is rarely seen in GISTs, is morphologically distinct from the classical GIST. The dedifferentiated component presents anaplastic/pleomorphic appearance,high nuclear atypia, high mitotic activity, and necrosis[3,4].The unusual histological and immunohistochemical characteristics of these tumors are challenging to diagnose. Therefore, pathologists must recognize GIST that has an abnormal shape and be aware of the process of dedifferentiation.

The most common symptoms of patients are bleeding, anemia, and abdominal pain. Other symptoms are also common, such as indigestion, nausea or vomiting, constipation, or diarrhea.[14,15,16]At first, our 
patient was hospitalized for anemia. We report a patient who received imatinib for 11 months after the discovery of a gastric stromal tumor by biopsy firstly囚and then had the tumor surgically removed. In the excised tumor specimens, we found that the classical mixed epithelioid and spindle cell GIST region was accompanied by a undifferentiated component of pleomorphic sarcoma.The genetic test results of the patients showed that the 11 exon of C-Kit gene was homozygous deletion, and the 567 codon of the 12 exon of PDGFR was homozygous mutation.

\section{Case Presentation}

The patient is a 64 - year - old Asian man, he was admitted to a local hospital for dizziness and anemia the past year,Computed tomography scan disclosed one large tumor at the bottom of the stomach $\otimes F I G 1 A, 1 B \otimes$, Subsequently, the endoscopic biopsy was performed, and the pathological diagnosis was "Gastrointestinal stromal tumors". The little tumor tissue was positive for CD117 and Dog-1 by

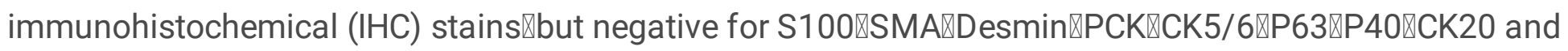
CDX-2ХDue to the large size of the tumor, the patient did not receive immediate surgical treatment, but began taking imatinib(400mg,qd), 11 months later, the patient was admitted to the local hospital for dizziness and fever, and then transferred to our hospital for further diagnosis and treatment. The outpatient diagnosis was "gastric stromal tumor with hemorrhage and anemia". Computed tomography scan disclosed the gastric wall of the fundus was significantly thickened, and a large mass with a size of $11.7 \mathrm{~cm} \times 0.7 \mathrm{~cm}$ was seen inside. The shape of the lesion was irregular, the edges of enhanced scanning were significantly enhanced, and large flaked areas of unenhanced liquefaction necrosis could be seen in the center $\otimes \mathrm{FIG} 2 \mathrm{~A}, \mathrm{~B} \bigotimes$. Then he underwent surgical treatment in our hospital, During the operation, the primary tumor was found to be located at the bottom of the stomach and grew out of the luminal cavity, involving the diaphragm and the tail of the pancreas. the gastric tumor, spleen and pancreas were removed, and the postoperative pathological diagnosis and genetic testing were performed. After the pathological diagnosis, imatinib administration was initiated $(400 \mathrm{mg} / \mathrm{d})$ at a few days after the operation, two months after the operation, the patient is alive with an outpatient status. At present, there are no serious postoperative complications.

\subsection{Materials and methods}

\subsubsection{Immunohistochemical analysis}

The surgical specimen was examined grossly and fixed in 10\% neutral formalin. The representative specimen was embedded in paraffin and submitted for permanent histological examination.Four-micron thick hematoxylin and eosin (H\&E) stained sections were prepared.

Immunohistochemical stains for CD117 (mouse monoclonal『Abcam), CD34 (mouse monoclonal, Ventana), DOG-1 ( rabbit monoclonal, Ventana), smooth muscle actin (SMA) (clone 1A4, mouse 


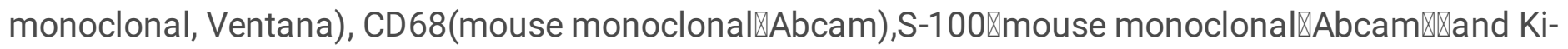
67(mouse monoclonal『Abcam) were performed by an automated immunostainer .

\subsubsection{Sequence analysis of the c-kit and PDGFRa gene}

For mutational analysis $\bowtie$ the paraffin-embedded sections of the patients' tumors were sent to guangzhou microread medical laboratory for detection of gene mutations associated with individualized treatment of tumors $邓$ the tissues examined included typical gastric stromal tumor and dedifferentiated pleomorphic sarcoma. The genomic DNA was extracted from paraffin-embedded sections of tumor tissue by using sureselect human all exon V6(Agilent,America),Genes (exons) analyzed include the following [Gene(exon)]:C-kit $(9,11,13,17)$ and PDGFRa $(12,18)$,The test was performed with the patient's informed consent.

\subsection{Results}

\subsubsection{Histopathology and immunohistochemistry}

The resection specimens showed that the tumor had separated from the gastric tissue. The size of the tumor was $15 \times 11 \times 4.5 \mathrm{~cm}$. The cut surface was grayish white and the local area was gray-red》 localized bleeding and necrosis can be seen on the incised surface.The tumor affected the spleen and pancreatic tissues. The gastric wall thickened to $1.1 \mathrm{~cm}$ immediately adjacent to the resection margin of esophagus, and the gastric mucosal surface at the thickening point is slightly rough. Microscopically, the tumor is composed of two distinct demarcated areas; one conventional GIST area and the other dedifferentiated area. The classic area consists mostly of epithelioid cells and spindle cells of uniform size, abundant cytoplasm, and rare nuclear pleomorphism. The tumor cells in classic GIST area was positive for CD117 (Fig. 3A),CD34 (Fig. 3B) and Dog-1 (focally,Fig. 3C) by immunohistochemical (IHC) stains. In particular, the dedifferentiated pleomorphic sarcoma component was found locally in the tumor, abruptly adjacent to the classical GIST. These pleomorphic cells are of various sizes, with large, hyperchromatic nuclei and prominent nucleoli, among which are bizarre multinucleated giant cells $\otimes F I G, 4)$. At higher magnification abnormal mitosis are easy to find. The undifferentiated pleomorphic sarcoma of the tumor was positive for SMA囚not shown掅ut negative for CD117 (Fig. 5A), CD34(Fig5B), DOG-1 (Fig. 5C), desmin (not shown), CD68(not shown), andS-100『not shown 囚by IHC stains. Notably, there was a loss of CD117and CD34 expression in the undifferentiated component of the tumor.

The resected pancreas, spleen and adrenal gland were involved by the tumor cells, and the live tumor showed a highly cellular and aggressive growth pattern.

Additionally, sarcomatoid tumor cells had higher mitotic activity by immunostain for proliferative index

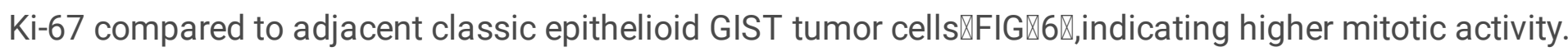


The resection margins were negative.After imatinib treatment, some areas of hyaline degeneration are seen in the tumor tissue(Fig. 7A).

\subsubsection{Mutational status of the patient}

We detected the exon 9/11/13/17 of c-kit and the exon 12/18 of PDGFRa gene, the patient's genetic test

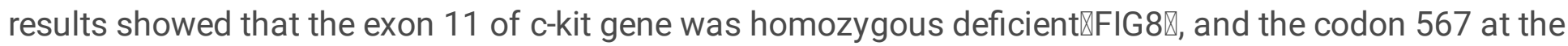

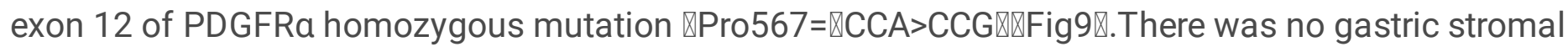
tumor in the immediate family. C-kit gene mutation is related to the efficacy of targeted therapy with imatinib, this test detected the 11 exon deletion mutation of c-kit gene. Patients with this mutation were more sensitive to imatinib treatment than patients with 9 exon mutation, and the treatment effect was the best.

\section{Discussion And Conclusions}

We encountered a case of gastric stromal tumor involving other abdominal organs, including spleen, pancreas and adrenal gland. His pathological diagnosis was classic GIST with undifferentiated pleomorphic sarcoma. Generally speaking, KIT and PDGFRA mutations are mutually exclusive[13],but the patient's genetic test results showed that the exon 11 of c-kit gene was homozygous deficient, and the codon 567 at the exon 12 of PDGFRa was homozygous mutation. The exon 12 of PDGFRa is a synonymous variant $\mathbb{\text { It }}$ is most likely a germline mutation.In this case, the significance of the mutation is unclear.The patient was given imatinib orally for 11 months (400mg, QD) prior to surgical treatment.

Until recently, dedifferentiated anaplastic variants of GISTs were mostly reported in patients who had received long-term treatment with the tyrosine kinase inhibitor imatinib mesylate [5,6,7].As in our case, in all other reported cases of dedifferentiation transformation of GIST, the prevalence of KIT (exon 11) and PDGFRa activating mutations was preserved in each tumor's dedifferentiated component, However, KIT protein expression (CD117) was either completely lost or significantly reduced with transformation [7, 8].In this case, after taking imatinib for 11 months, the resected specimen showed obvious degeneration of tumor tissue, Although the abrupt transition from CD117-positive classic GIST to CD117-negative undifferentiated pleomorphic sarcoma is striking, the underlying molecular mechanism remains uncertain. New dedifferentiated GISTs have only been reported in a handful of cases. It has been shown that dedifferentiation can occur through KIT-independent mechanisms with loss of KIT expression and altered morphology $[9,10,11]$.In addition to gross chromosomal rearrangements, potential alterations could include mutations and/or small insertions/deletions that disrupt the open reading frame of the KIT gene or the splicing and processing of the KIT mRNA. Additionally, mutations within the KIT promoter may prevent transcription of the gene, preventing KIT protein expression[12].

It is clinically significant to note that dedifferentiation mostly occurred in metastatic or recurrent lesions[5.7.10].Antonescu et al. investigated the underlying molecular mechanism of tumor progression in three imatinib-resistant and five imatinib-naïve tumors. Molecular characterization of the tumors 
showed that half of them had wild type KIT, PDGFRA, and BRAF genes in both conventional and dedifferentiated components in contrast to KIT-mutant GIST. These findings suggest that dedifferentiation can be triggered through alternative escape mechanisms besides activating mutations. Loss of a KIT gene copy due to haplo-insufficiency was found in the dedifferentiated components of the three KIT-negative imatinib-resistant GISTs. Two imatinib-resistant tumors showed co-existence of KIT mutations in exons 11 and 13[10] .

In this case, the patient's initial pathological diagnosis showed no dedifferentiated components, but it may also be caused by limited sampling. We prefer patients with dedifferentiation changes after imatinib administration, and the genetic changes are consistent with those reported in other cases. The mechanism of this change is still unclear. Related research shows that the dedifferentiation of GISTs can occur after a long time treatment with Imatinib and presents with different morphologies, or rhabdomyosarcoma, angiosarcoma, or undifferentiated pleomorphic sarcoma.

In summary, the case shows morphologic and immunophenotypic pleomorphic sarcoma dedifferentiation of a treatment refractory GIST , with hyaline changes of tumor cells after IM therapy. Together with reported cases, awareness of this rare clinical entity and its potential occurrence following Tyrosine kinase inhibitor (TKI)treatment could prevent a diagnostic pitfall. The case report reminds us of the diagnosis in GIST that the possibility of dedifferentiation in GISTs should always be considered when an undifferentiated sarcoma component is identified in the gastrointestinal tract. Molecular analysis provides valuable information exploring tumor origin and tumor progression and may assist with optimal treatment strategies in the future.

\section{Declarations}

\section{- Ethics approval and consent to participate}

The article did not disclose any patient's personal privacy, and was written with the patient's informed consent, without any ethical issues.

\section{- Consent for publication}

No conflict of interest exists in the submission of this manuscript, and the manuscript is approved by all authors for publication.

\section{- Availability of data and materials}

Not applicable

\section{- Competing interests}

The authors declare that they have no competing interests 


\section{- Funding}

No funding

\section{- Authors' contributions}

Yifanhu analyzed and explained the patient's diagnosis, medical history and other relevant data, and was the main author of the paper.Shunhaijian, who conducted histological examinations of patient tissue, was a major contributor to the manuscript and provided guidance on editing and writing articles.All authors read and approve the final manuscript.

\section{- Acknowledgements}

Not applicable

\section{References}

1. A T, et al. [A Case of Esophageal Submucosal Tumor Undiagnosed Preoperatively and Treated with Thoracoscopic Enucleation]. Gan to kagaku ryoho. Cancer chemotherapy. 2016;43(12):2175-7.

2. Antonescu CR. The GIST paradigm: lessons for other kinase-driven cancers. J Pathol. 2011;223(2):251-61.

3. Antonescu CR, Romeo S, Zhang L, Nafa K, Hornick JL, Nielsen GP, et al.Dedifferentiation in stromal tumor to an anaplastic kit-negativePhenotype: a diagnostic Pitfall: morphologic and molecular characterization of 80ccurring in either DE novo or after imatinib therapy. Am J Surg Pathol2013;37 (3): $385-92$.

4. Jiang X, Anderson HB, Guy CD, Mosca PJ, Riedel RF, Cardona DM. Rhabdomyosarcomatous transformation of a gastrointestinal stromal tumor following treatment with imatinib. Case Rep Oncol Med. 2015;2015:317493.

5. Pauwels P, Debiec-Rychter M, Stul M, De Wever I, Van Oosterom AT, Sciot R. Changing phenotype of gastrointestinal stromal tumours under imatinib mesylate treatment: a potential diagnostic pitfall. Histopathology. 2005;47(1):41-7.

6. Diaz Delgado M, Hernandez Amate A, Pereira Gallardo S, Jaramillo S, Virizuela Echaburu JA, Gonzalez-Campora RJ. Gastrointestinal stromal tumors: morphological, immunohistochemical and molecular changes associated with kinase inhibitor therapy. Pathol Oncol Res. 2011;17(3):455-61.

7. Liegl B, Hornick JL, Antonescu CR, Corless CL, Fletcher CD. Rhabdomyosarcomatous differentiation in gastrointestinal stromal tumors after tyrosine kinase inhibitor therapy: a novel form of tumor progression. Am J Surg Pathol. 2009;33(2):218-26.

8. Zhu P, Fei Y, Wang Y, Ao Q, Wang G. Recurrent retroperitoneal extra-GIST with rhabdomyosarcomatous and chondrosarcomatous differentiations: a rare case and literature review. Int J Clin Exp Pathol. 2015;8(8):9655-61. 
9. Choi JJ, Sinada-Bottros L, Maker AV, Weisenberg E. Dedifferentiated gastrointestinal stromal tumor arising de novo from the small intestine. Pathol Res Pract. 2014;210(4):264-6.

10. Antonescu CR, Romeo S, Zhang L, Nafa K, Hornick JL, Nielsen GP, et al. Dedifferentiation in gastrointestinal stromal tumor to an anaplastic KIT-negative phenotype: a diagnostic pitfall: morphologic and molecular characterization of 8 cases occurring either de novo or after imatinib therapy. Am J Surg Pathol. 2013;37(3):385-92.

11. Jung JH, Im S, Choi HJ, Lee YS, Jung ES. Gastrointestinal stromal tumor with dedifferentiation to undifferentiated pleomorphic sarcoma. Pathol Int. 2013;63(9):479-82.

12. $L \mathrm{~L}$, et al. Case report of rhabdomyosarcomatous transformation of a primary gastrointestinal stromal tumor (GIST). BMC Cancer. 2019;19(1):913.

13. BP R, H. MC and CL C. Gastrointestinal stromal tumour. Lancet. 2007;369(9574):1731-41.

14. Muccariani C, Rossi G, Bertolini F, et al. Incidence and clinicopathologic features of gastrointestinal stromal tumors. A population-based study. BMC Cancer. 2007;7:230.

15. Caterino S, Lorenzon L, Petrucciani N, et al. Gastrointestinal stromal tumors: correlation between symptoms at presentation, tumor location and prognostic factors in 47 consecutive patients. World $\mathrm{J}$ Surg Oncol. 2011;9:13.

16. Bümming P, Ahlman H, Andersson J, Meis-Kindblom JM, Kindblom LG, Nilsson B. Population-based study of the diagnosis and treatment of gastrointestinal stromal tumours. Br J Surg. 2006;93:83643.

\section{Figures}
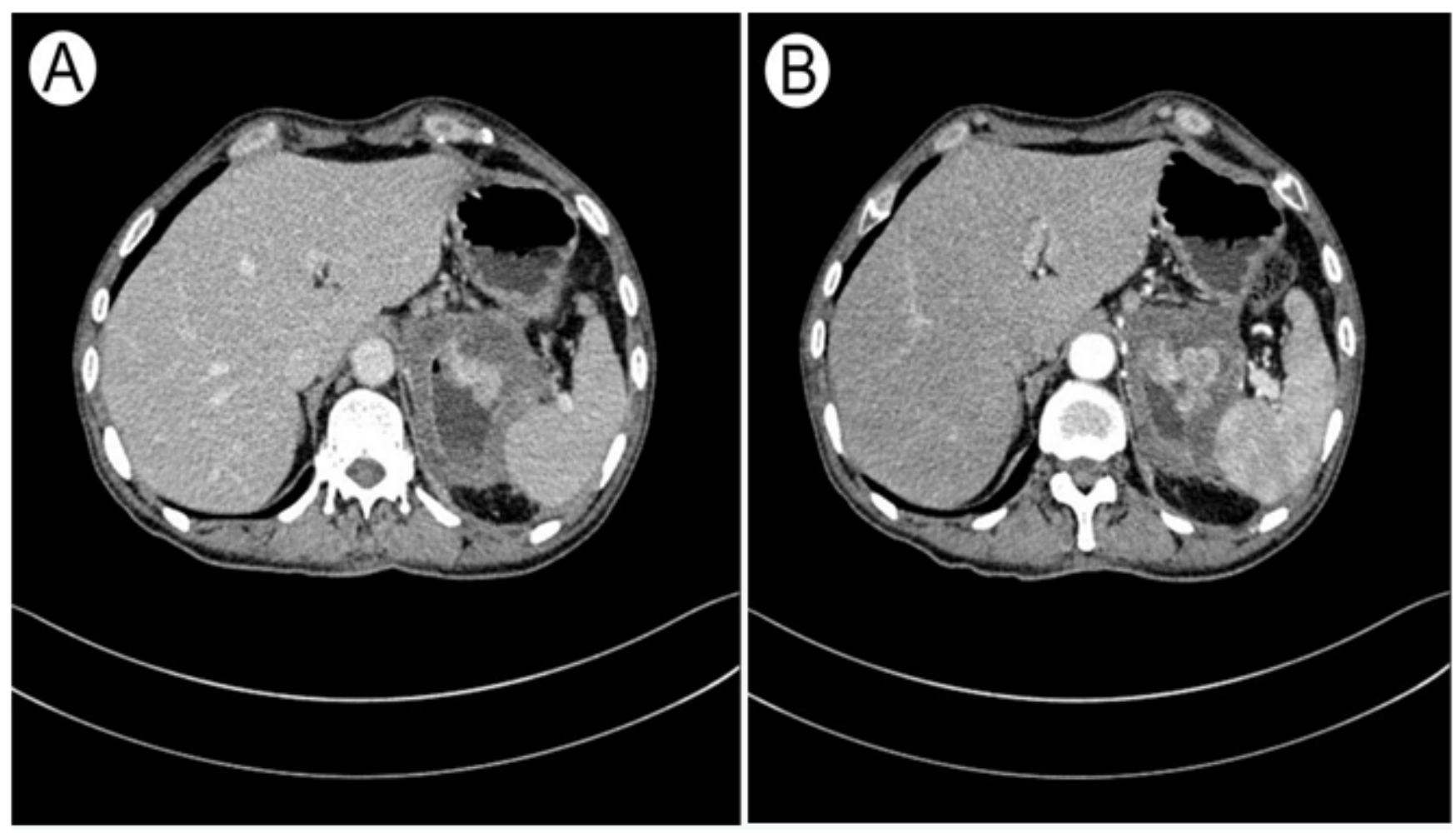


\section{Figure 1}

Ct scan of gastrointestinal stromal tumors (GIST) before imatinib administration: A Ordinary scanning $₫ \mathrm{~B}$ enhanced scanning

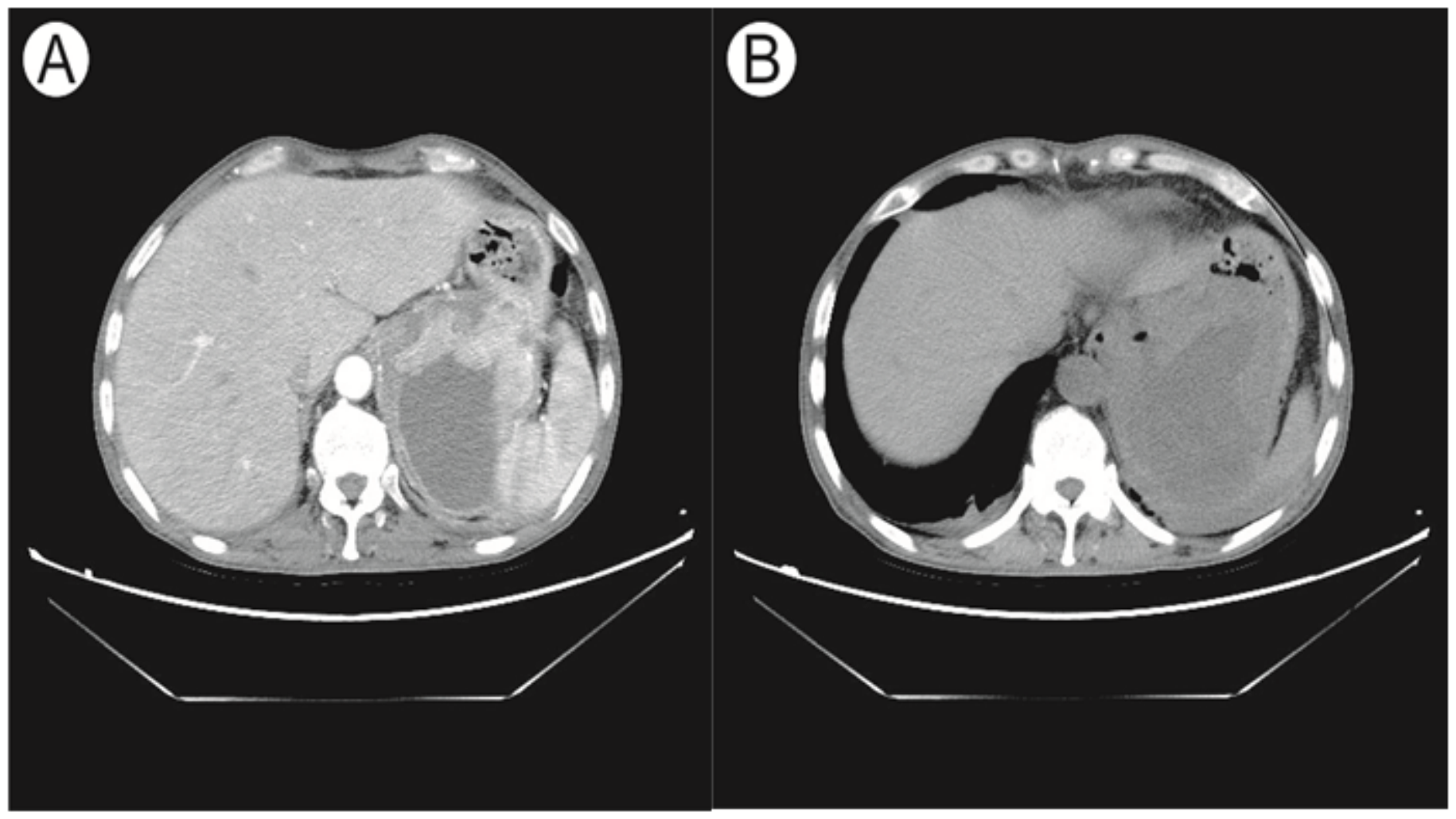

Figure 2

2CT-scan of Gastrointestinal Stromal Tumor (GIST) Prior to Surgery: A enhanced scanning $₫ \mathrm{~B}$ Ordinary scanning 

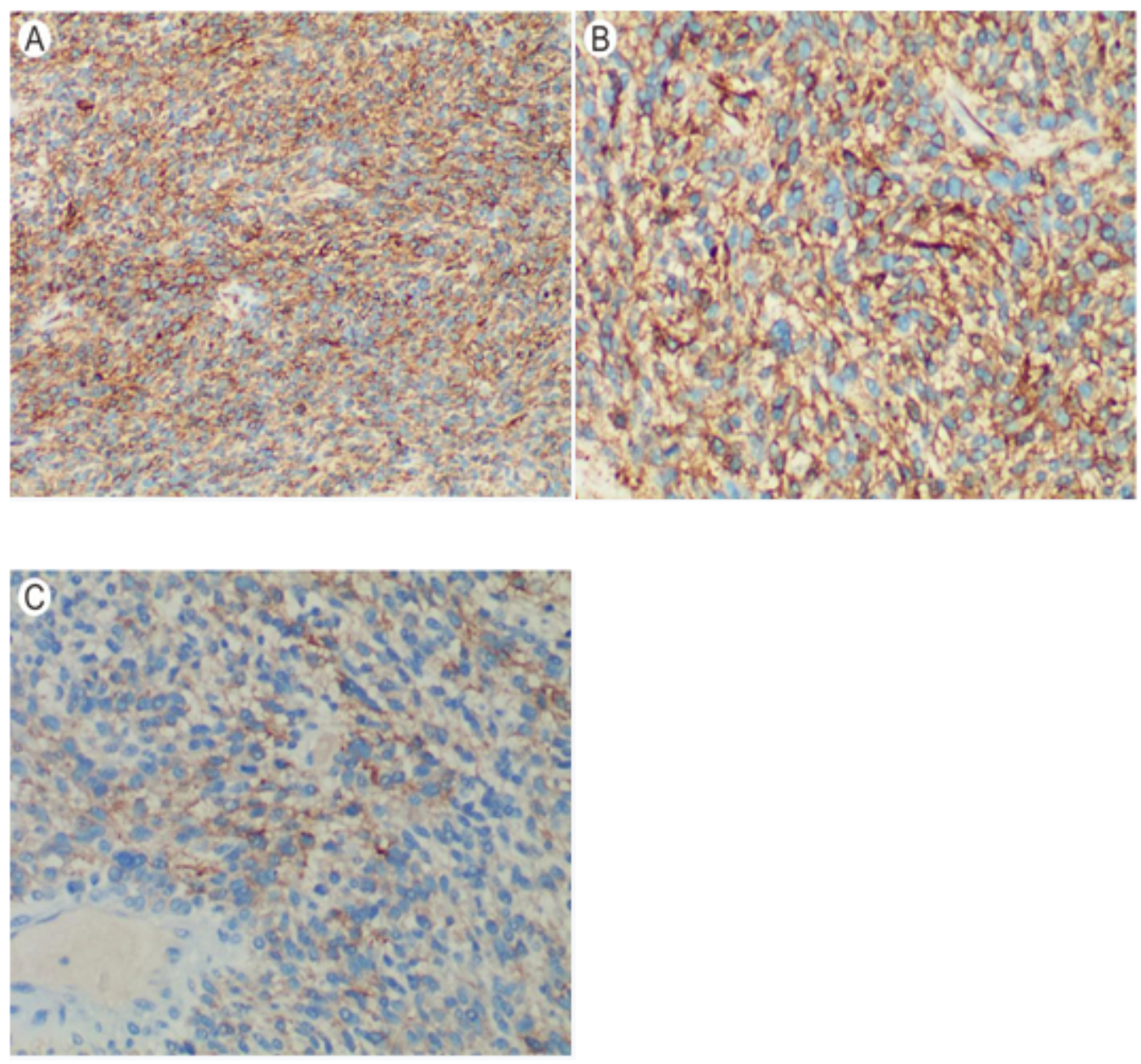

\section{Figure 3}

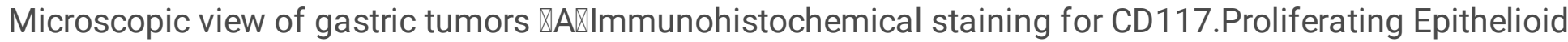
cells were positive for CD117区B:Immunohistochemical staining for CD34. Proliferating Epithelioid cells were positive for CD34,C:Immunohistochemical staining forDog-1. Proliferating Epithelioid cells were FOCALLY positive for Dog-1. 


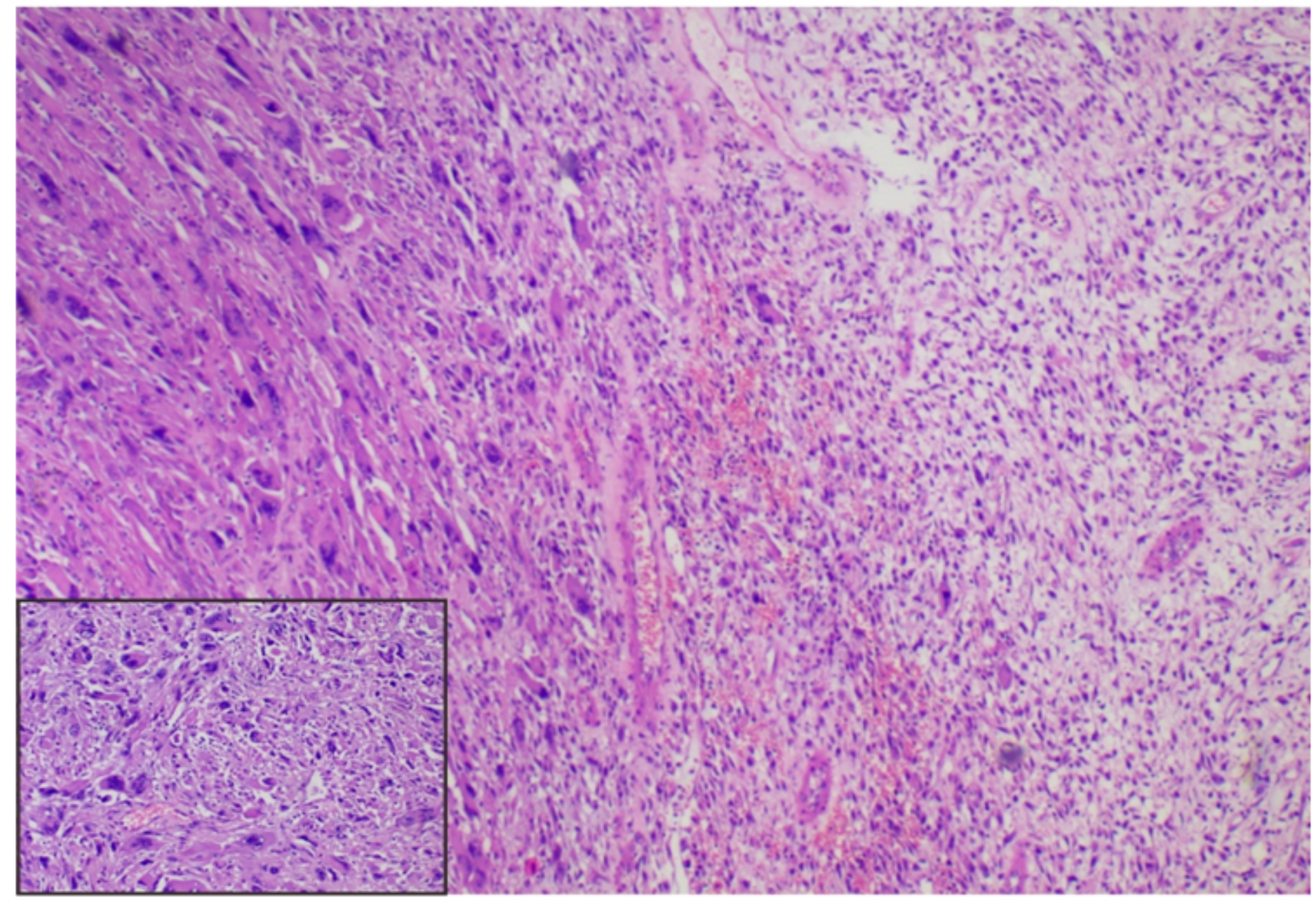

\section{Figure 4}

Microscopic view of resected tumor,Hematoxylin-eosin staining $₫$ the undifferentiated pleomorphic sarcoma component was found locally in the tumor, abruptly adjacent to the classical epithelioid GIST》 These pleomorphic cells are of various sizes, with large, hyperchromatic nuclei and prominent nucleoli, among which are bizarre multinucleated giant cells.

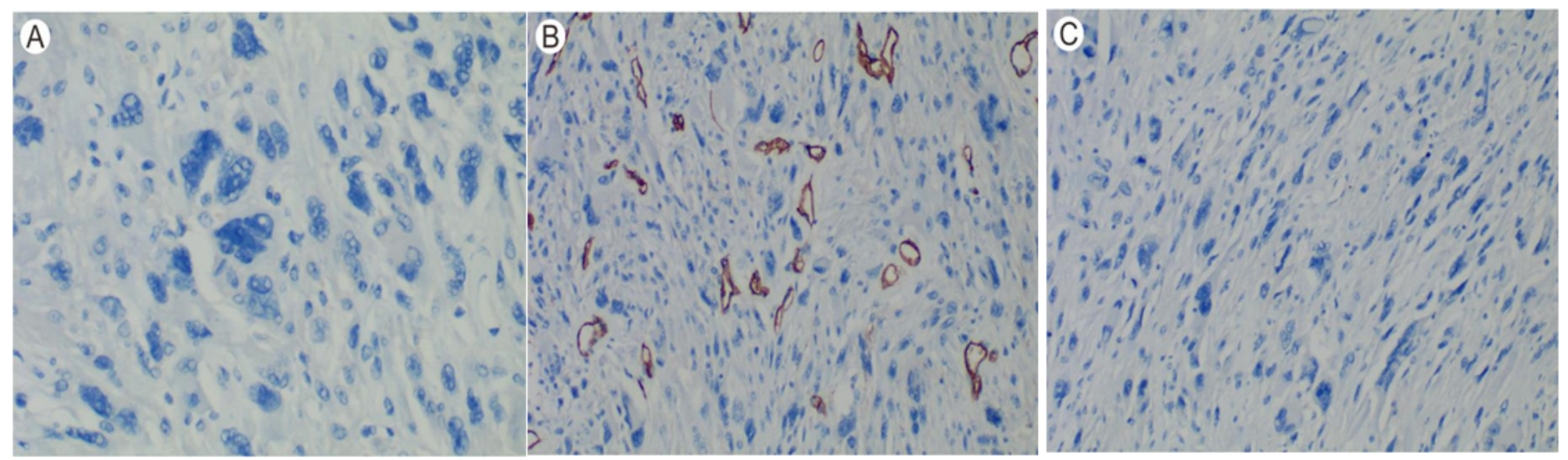

Figure 5 
Microscopic view of gastric tumor,A:Immunohistochemical staining for CD117.the undifferentiated component of the tumorwere negative for CD117,B:Immunohistochemical staining for CD34.the undifferentiated component of the tumorwere negative for CD34,C:Immunohistochemical staining for Dog-1,the undifferentiated component of the tumorwere negative for Dog-1.

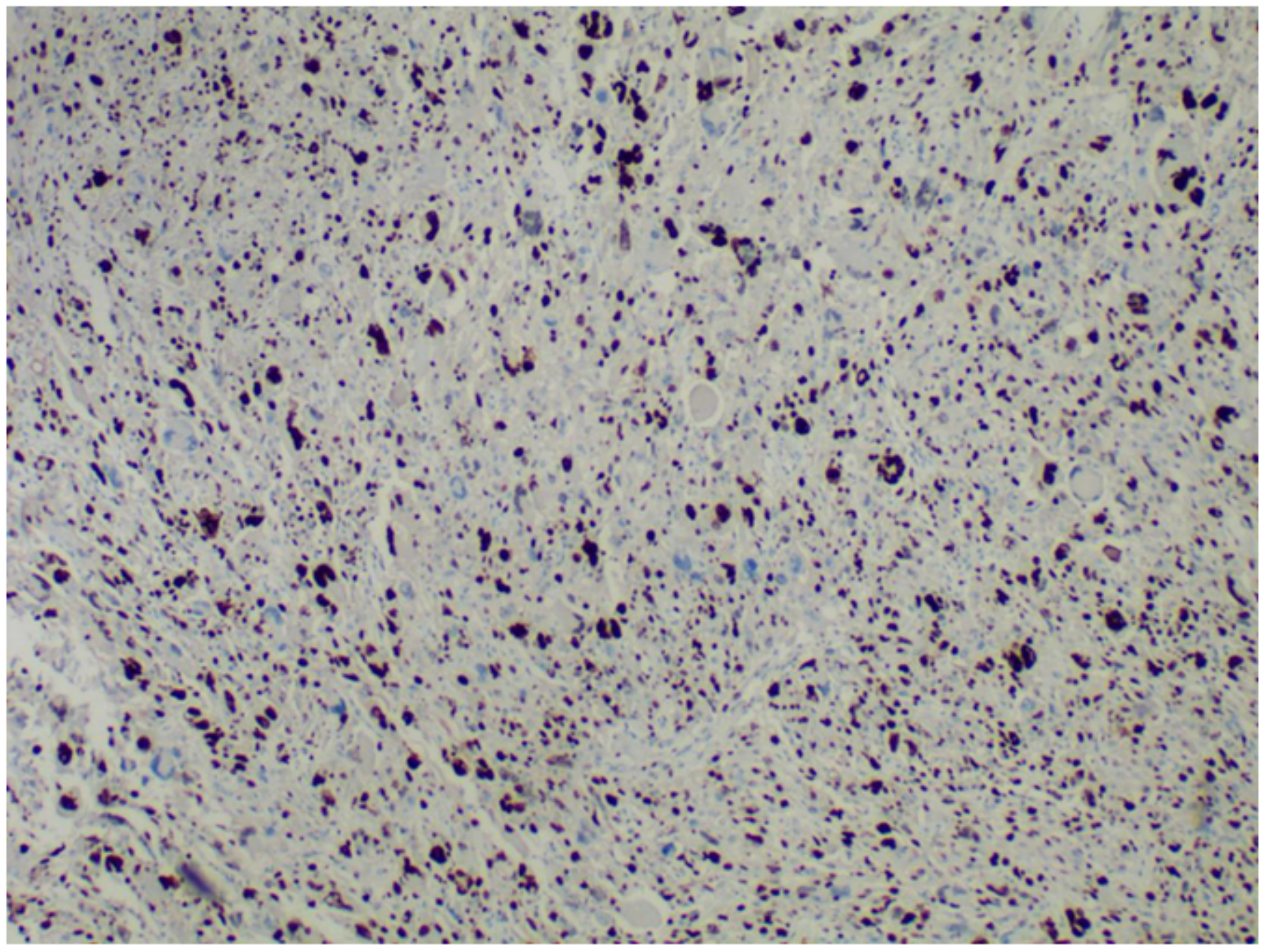

Figure 6

Microscopic view of the undifferentiated component in the gastric tumor ,Immunohistochemical staining for Ki-67,the high proliferative activity of the components of an undifferentiated pleomorphic sarcoma is shown here. 


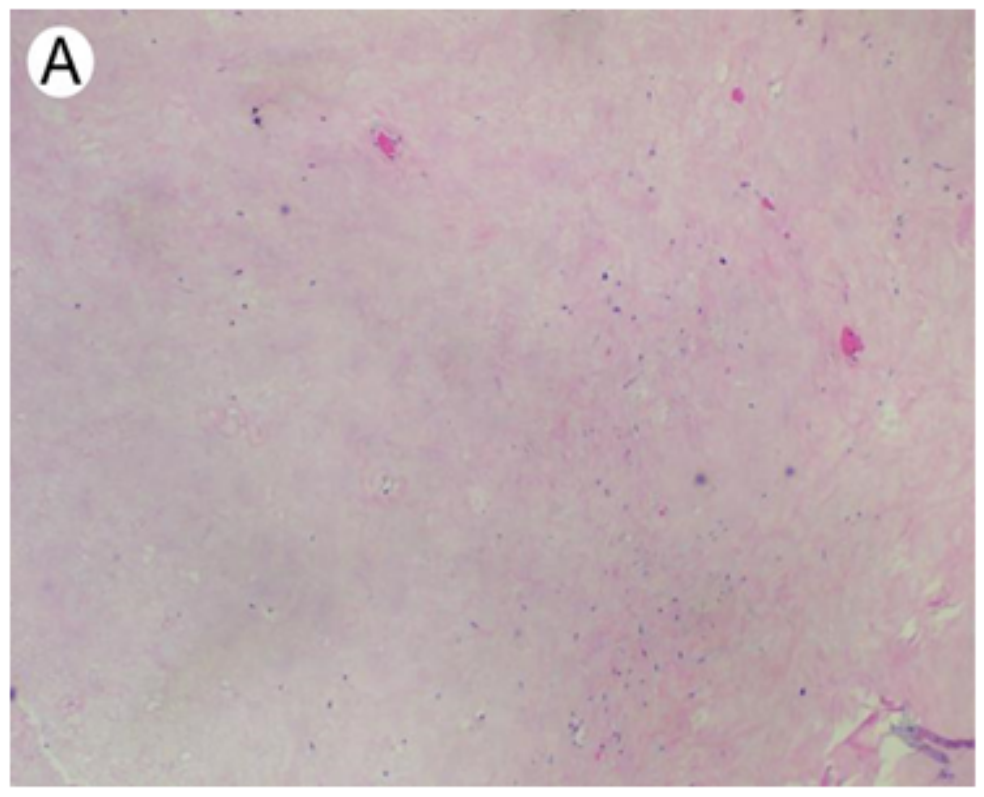

Figure 7

Microscopic view of gastric tumor冈hyaline degeneration.

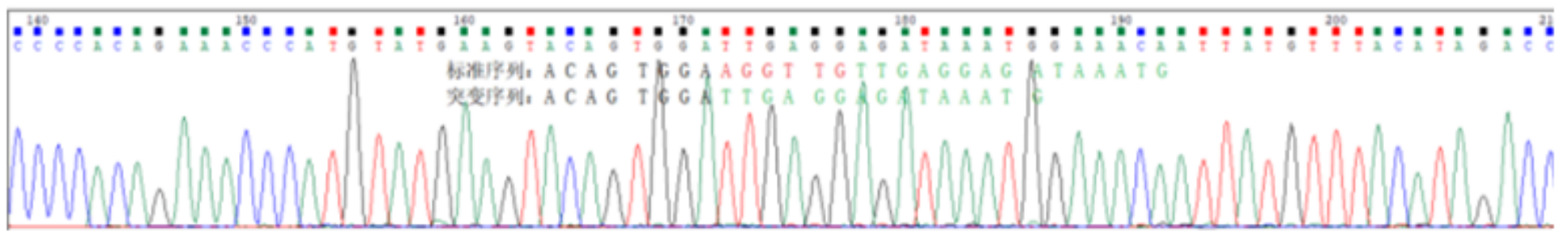

Figure 8

Result of sequencing analysis at exon 11 of the c-kit gene.

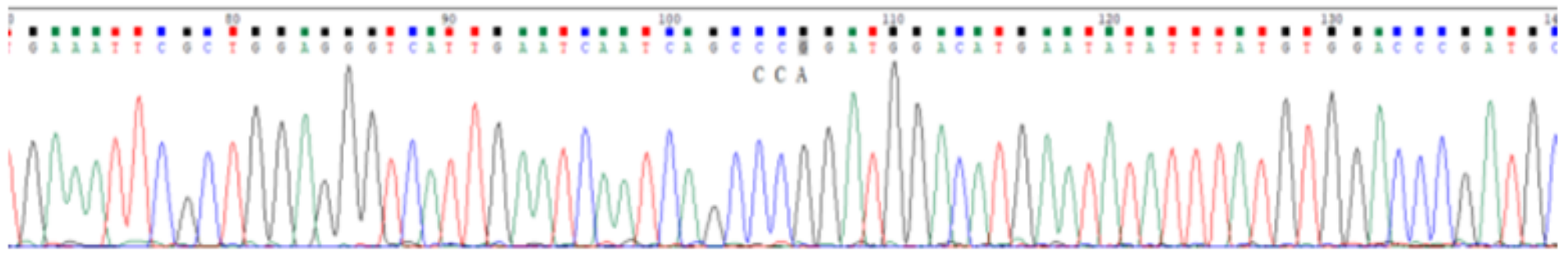

Figure 9

Result of sequencing analysis at exon 12 of PDGFRa

\section{Supplementary Files}

This is a list of supplementary files associated with this preprint. Click to download.

- renamedffdb6.doc 